\title{
IMPACTO DA SECAGEM CONVECTIVA SOBRE OS COMPOSTOS BIOATIVOS E ATIVIDADE ANTIOXIDANTE DO RESÍDUO DE CAJU (Anacardium Occidentale L.)
}

\author{
F. P. MORAES ${ }^{1}$, E. S. SILVA ${ }^{1}$; P. M. ROCHA ${ }^{1}$; T. R. N. FERNANDES ${ }^{1}$; R. H. L. VIDAL ${ }^{1}$ \\ R. T. P. CORREIA ${ }^{1}$
}
${ }^{1}$ Universidade Federal do Rio Grande do Norte, Departamento de Engenharia Química E-mail para contato: franciscapereira.moraes@gmail.com

\begin{abstract}
RESUMO - O processamento industrial do caju gera volumes consideráveis de resíduos agroindustriais e uma das alternativas de aproveitamento desse resíduo é a secagem convectiva, técnica flexível e de baixo custo. Dessa forma, o presente trabalho avalia o impacto da secagem convectiva a $80^{\circ} \mathrm{C}$ sobre os compostos fenólicos totais (método de Folin-Ciocalteu), ácido ascórbico (titulometria), carotenoides (espectrofotometria) e atividade antioxidante (radical DPPH) do resíduo de caju. Os valores de retenção foram calculados tendo o resíduo in natura como grupo controle. O resíduo de caju mostrou considerável retenção nos teores de fenólicos $(52,4 \%)$, carotenoides $(75,0 \%)$ e atividade antioxidante (60,2\%), mas o ácido ascórbico sofreu significante impacto da secagem e apresentou retenção de apenas $24,9 \%$. Apesar das perdas decorrentes do processo, o resíduo de caju seco apresentou elevado teor residual de compostos bioativos, o que demonstra sua potencialidade como ingrediente funcional para a formulação de alimentos. Palavras chave: resíduo de caju, impacto do processamento, secagem convectiva.
\end{abstract}

\section{INTRODUÇÃO}

O Brasil se destaca no ranking mundial como o terceiro maior produtor de frutos (FAO, 2012) e o quarto maior produtor de caju (Anacardium occidentale L.), sendo grande parte dessa produção concentrada na região Nordeste (Cartilha, 2010).

O caju é constituído pela castanha, verdadeiro fruto do cajueiro, e pelo pseudofruto ou pedúnculo. Esse último apresenta coloração amarela pálida, carnosa, suculenta, sabor ácido adstringente, além de aroma característico (Chitarra e Chitarra, 2005). Muito apreciado por seus atributos sensoriais e nutricionais (Infante et al. 2013), o caju apresenta alta perecibilidade. Dessa forma, além do consumo in natura, é processado na forma de produtos derivados: polpas, sucos concentrados, néctares, entre outros (Souza Filho et al. 2000). 
O processamento de frutas na indústria gera volume considerável de resíduos (casca, sementes e bagaço), o que acarreta em potencial problema ambiental. A ausência de estratégias para o correto aproveitamento desse material leva a perdas de matéria e energia, além do desperdício de produtos com elevado conteúdo bioativo. Nesse contexto, há a necessidade do desenvolvimento de programas e/ou tecnologias de reutilização desses resíduos que promovam a conversão do descarte da agroindústria em produtos de valor agregado, como biocombustível, suplementos alimentares e outros (Makris et al. 2007; Roriz, 2012).

Dentre as tecnologias que podem ser empregadas para o reaproveitamento e utilização dos resíduos está à secagem convectiva (Ferreira e Pena, 2010; Perazzini, 2011; Prette, 2012; Nóbrega et al. 2014). Além de reduzir a umidade do produto e promover maior estabilidade ao produto final, essa técnica se caracteriza por seu baixo custo e flexibilidade (Singh e Heldman, 2001; Park et al., 2007; Prette, 2012).

Sendo assim, o objetivo do presente trabalho foi avaliar o impacto da secagem convectiva sobre o resíduo de caju. Para isso, a retenção do teor de ácido ascórbico, compostos fenólicos, carotenoides e atividade antioxidante são apresentados e discutidos. Os resultados permitem avaliar a adequação da aplicação dessa técnica ao resíduo de caju, bem como revela o valor bioativo do resíduo de caju desidratado por convecção.

\section{MATERIAL E MÉTODOS}

\subsection{Preparo das amostras}

O pedúnculo de caju foi obtido no mercado local (Natal, RN). Após o processamento da polpa, o resíduo in natura obtido foi mantido congelado até o momento do uso. O resíduo de caju foi submetido ao processo de secagem para obtenção de pós utilizando secador convectivo de bandejas a temperatura de $80{ }^{\circ} \mathrm{C}$, velocidade do ar $6 \mathrm{~m} / \mathrm{s}$ e o tempo de 300 minutos.

\subsection{Elaboração dos extratos aquosos}

Para a elaboração do extrato, pesou-se $1 \mathrm{~g}$ da amostra, a qual foi homogeneizada em $50 \mathrm{~mL}$ de água destilada sob agitação por $1 \mathrm{~h}$. A solução foi filtrada e centrifugada a 3600 rpm por $10 \mathrm{~min}$. O sobrenadante foi mantido refrigerado até o momento da análise. O extrato foi utilizado nas análises de compostos fenólicos totais e atividade antioxidante. Os demais métodos foram conduzidos mediante pesagem direta da amostra.

\subsection{Análises}

O teor de compostos fenólicos totais, carotenoides, teor de ácido ascórbico e atividade antioxidante foram avaliados no resíduo de caju in natura e no desidratado. As análises foram realizadas em triplicata, empregando-se os métodos analíticos descritos a seguir. Com o objetivo de avaliar o impacto da secagem, os resultados foram expressos em base seca (bs). 


\subsubsection{Determinação de compostos fenólicos}

A determinação do teor de compostos fenólicos totais (CFT) foi conduzida pelo método de Folin-Ciocalteau de acordo com Nóbrega et al. (2014). Os resultados foram expressos como mg ácido gálico equivalente (GAE)/ $100 \mathrm{~g}$ bs.

\subsubsection{Teor de ácido ascórbico}

Para o teor de ácido ascórbico (AA) utilizou-se o método titulométrico do 2,6diclorofenolindofenol (DCFI) descrito por Oliveira et al. (2010) utilizando ácido metafosfórico como solução extratora. O teor de AA foi expresso em mg/100 g bs.

\subsubsection{Carotenoides}

$\mathrm{Na}$ determinação dos carotenoides, foi utilizada a metodologia descrita por Lichtenthaler e Buschmann (2001) e os resultados foram expressos em $\mu \mathrm{g} / \mathrm{g}$ bs.

\subsubsection{Atividade antioxidante}

A atividade antioxidante foi avaliada através da redução do radical estável DPPH• (2,2- difenil-1-picrilhidrazil) segundo método descrito por Brand-Williams et al. (1995) e modificada por Duarte-Almeida et al. (2006), Os resultados foram expressos em micromoles de Trolox equivalente (TE) por grama de amostra em base seca ( $\mu \mathrm{mol} \mathrm{TE} / \mathrm{g} \mathrm{bs})$.

\subsubsection{Cálculo da retenção}

A retenção dos compostos bioativos e da atividade antioxidante foi avaliada de acordo com Nóbrega et al. (2014) pela relação do conteúdo final e inicial expressos em base seca (Equação 1):

Retenção $(\%)=\frac{\text { A final }}{\text { A inicial }} * 100$

Onde:

$\mathrm{A}_{\text {inicial }}=$ teor do componente no resíduo in natura (bs);

$\mathrm{A}_{\text {final }}=$ teor do componente após a secagem convectiva (bs).

\subsubsection{Análise estatística}

O tratamento estatístico dos dados se deu pela análise de variância (ANOVA) e Teste de Tukey, utilizando o software Statistica 7.0 (Statsoft) com diferença estatística de 5\% ( $\mathrm{p}<$ $0,05)$. Os resultados foram exibidos como média \pm desvio padrão $(n=3)$. 


\section{RESULTADOS E DISCUSSÃO}

Na Tabela 1, estão expressos os resultados obtidos para os compostos bioativos (compostos fenólicos totais, ácido ascórbico e carotenoides) e a atividade antioxidante encontrado nos resíduos de caju in natura e desidratado por secagem convectiva expressos em base seca.

Tabela 1 - Concentração de ácido ascórbico, compostos fenólicos totais, carotenoides e atividade antioxidante nos resíduos de caju in natura (RN) e desidratado (C) expressos em base seca.

\begin{tabular}{|lcccc|}
\hline & $\begin{array}{c}\text { Compostos fenólicos } \\
(\mathbf{m g} \text { GAE / 100g bs })\end{array}$ & $\begin{array}{c}\text { Carotenoides } \\
(\boldsymbol{\mu g} / \mathbf{g} \text { bs })\end{array}$ & $\begin{array}{c}\text { Ácido ascórbico } \\
(\mathbf{m g} / \mathbf{1 0 0 g} \mathbf{b s})\end{array}$ & $\begin{array}{c}\text { Atividade antioxidante } \\
\mathbf{D P P H}(\boldsymbol{\mu m o l ~ T E} / \mathbf{g} \text { bs })\end{array}$ \\
\hline $\mathbf{R N}$ & $517,00 \pm 8,61^{\mathrm{a}}$ & $121,20 \pm 3,75^{\mathrm{a}}$ & $859,77 \pm 1,03^{\mathrm{a}}$ & $35,84 \pm 4,19^{\mathrm{a}}$ \\
\hline $\mathbf{C}$ & $271,06 \pm 4,2^{\mathrm{b}}$ & $90,88 \pm 2,10^{\mathrm{b}}$ & $213,62 \pm 0,09^{\mathrm{b}}$ & $21,57 \pm 1,95^{\mathrm{b}}$ \\
& & & & \\
\hline
\end{tabular}

Média \pm desvio padrão $(n=3) ;{ }^{a, b}$ médias seguidas de letras diferentes em cada linha são significativamente diferente $(\mathrm{p}<0,05)$. TE: trolox equivalente. bs- base seca.

Os compostos fenólicos são metabólitos secundários das plantas, que apresentam capacidade de combater os radicais livres, atuando na prevenção de doenças crônicas (Haida et al. 2011; Melo et al. 2011). Os resultados dos CFT dos resíduos de caju in natura e desidratado (Tabela 1) mostram que essas amostras são estatisticamente diferentes $(\mathrm{p}<0,05)$. A retenção observada $(52,4 \%)$ revela a magnitude das perdas causadas pela secagem. No entanto, o resíduo seco ainda apresenta boa quantidade de fenólicos, superior ao teor encontrado por Nóbrega et al. (2014) no resíduo de acerola (na faixa de 2,69 - 3,17 mg GAE eq/100 g b.s.) obtido por secagem convectiva, o que resultou na retenção de $31,7 \%$ desses constituintes.

Infante et al. (2013) avaliando os compostos fenólicos em resíduos de frutos observaram que o teor de fenólicos das frutas analisadas obedeceu a ordem caju > manga > maracujá > abacaxi. Os mesmos autores obtiveram menores valores de CFT no resíduo de caju liofilizado (10,67 mg GAE/g bs) quando comparado ao resíduo desidratado do presente estudo. Roy et al. (2007) citam que o processo de aquecimento a temperaturas mais brandas preservam melhor o teor de compostos fenólicos presentes nas amostras.

Os carotenoides são pigmentos lipossolúveis que conferem as cores amarela, laranja e vermelha a diversos vegetais (Rodriguez-Amaya et al. 2008; Almeida et al. 2009). O resíduo in natura mostrou valores superiores aos encontrados por Almeida et al. (2009) em polpa de acerola $(20,73 \mu \mathrm{g} / \mathrm{g})$ e Barreto et al. (2007) no bagaço de caju in natura $(43,3 \mu \mathrm{g} / \mathrm{g})$. A comparação dos valores obtidos antes e depois da secagem convectiva a $80{ }^{\circ} \mathrm{C}$ revela retenção de $75,0 \%$. Esse resultado é superior ao encontrado por Hiane et al. (2003) na farinha polpa de bacuri (retenção de 63\%) obtida pelo processo de desidratação em estufa. 
O ácido ascórbico (AA), forma ativa da vitamina C, é um composto antioxidante, termolábil e hidrossolúvel, amplamente presente em produtos de origem vegetal. O teor de AA depende de vários fatores, tais como época de colheita, variações genéticas, além das condições de processamento e armazenamento (Hassimotto et al. 2005. Barcia et al. 2010). Os resultados da Tabela 1 permitem identificar que a secagem convectiva repercute em significante diferença $(\mathrm{p}<0,05)$ entre as amostras de resíduo de caju in natura e seco. A retenção de 24,9\% revela o impacto negativo causado pela técnica de secagem utilizada aqui. Os dados confirmam a termolabilidade do AA ao processo de secagem convectiva. Previamente, Nóbrega et al. (2014) avaliaram o impacto da secagem convectiva sobre o teor de AA do resíduo de acerola e obtiveram valores de retenção de 76,3\%, superior ao encontrado aqui. Vale salientar que no presente estudo, fez-se uso de temperatura moderada de secagem de $80{ }^{\circ} \mathrm{C}$, a qual consequentemente acarreta em maior tempo de secagem e maior exposição da amostra ao ar, fatores considerados nocivos à manutenção do AA (Zerdin et al. 2003; Tanaka, 2007; Torales et al. 2008; Nóbrega et al. 2014).

O método utilizado para determinar a atividade antioxidante neste trabalho se baseia na capacidade de substâncias antioxidantes presentes no substrato reagirem com o radical livre DPPH• (Duarte-Almeida et al. 2006). O resíduo seco apresentou boa atividade antioxidante (Tabela 1), sendo esta influenciada pela presença de compostos fenólicos, ácido ascórbico e carotenoides, constituintes presentes em quantidades consideráveis no resíduo seco (Rocha et al., 2011; Valdés et al., 2012; Vinha et al., 2014).

A atividade antioxidante apresentada pelo resíduo desidratado foi menor que a encontrada por Infante et al. (2013) para resíduo de caju liofilizado (68,60 $\mu \mathrm{g}$ de TE/g bs). Mesmo apresentando modesta atividade antioxidante quando comparado a outros resíduos tais como manga (33,03 $\mu \mathrm{g}$ de TE/g bs) e bagaço de uva (116,02 e 389,9 $\mu \mathrm{g}$ de TE/g bs), o resíduo desidratado de caju ainda reteve 60,2\% dessa atividade (Infante et al, 2013; Cataneo et al., 2008; Melo et al. 2008). Dessa forma, apesar das perdas observadas, é possível inferir que a secagem convectiva produz produto final com boa concentração de compostos bioativos e propriedades antioxidantes.

\section{CONCLUSÕES}

De acordo com os resultados apresentados, podemos concluir que tanto o resíduo do pedúnculo de caju in natura, quanto o resíduo desidratado contém em sua composição quantidades relevantes de compostos bioativos. Dentre os fitoquímicos presentes nos resíduos destacam-se os carotenoides e os compostos fenólicos, os quais mostraram maior retenção após o processo de secagem. Evidencia-se, portanto, que a secagem convectiva aplicada ao resíduo do pedúnculo é uma estratégia que possibilitaria maior aproveitamento desse coproduto da indústria de caju, o qual diminuiria a potencial contaminação ambiental que o excesso desses resíduos vem causando. 


\section{REFERÊNCIAS}

ALMEIDA, C. B.; MANICA-BERTO, R.; FRANCO, J. J. PEGORARO, C.; FACHINELLO, J. C.; SILVA, J. A. Comparação do teor de carotenóides em frutos nativos de regiões tropicais e temperadas. XVIII Congresso de Iniciação Científica. Pelotas, RS, 2009.

BARCIA, M. T.; JACQUES, A. C.; PERTUZATTI, P. B.; ZAMBIAZI, R. C. Determinação de ácido ascórbico e tocoferóis em frutas por CLAE. Semana: Ciências Agrárias, Londrina, v. 31, n. 2, p. 381-390, abr./jun. 2010.

BARRETO, G.P.M.; SOUZA, A.C.R.; AZEREDO, H.M.C.; MERCADANTE, A.Z. Bioactive compounds and free radical scavenger activity in ingredients prepared from the waste of the cashew-apple nut industry. Alimentos e nutrição, v.18, n.2, p. 207-213, 2007.

BRAND-WILLIAMS, W., CUVELIER, M. E., BERSET, C. Use of a free radical method to evaluate antioxidant activity. LWT - Food Science and Technology, 28, p.25-30, 1995.

CARTILHA. Fruticultura-caju-Desenvolvimento Regional Sustentável, Série cadernos de propostas para atuação em cadeias produtivas, vol. 4, Banco do Brasil, setembro, 2010.

CATANEO, C. B.; CALIARI, V.; GONZAGA, L. V.; KUSKOSKI, E. M.; FETT, R. Atividade antioxidante e conteúdo fenólico do resíduo agroindustrial da produção de vinho. Semina: Ciências Agrárias, Londrina, v. 29, n. 1, p. 93-102, jan./mar. 2008.

CHITARRA, M. I. F.; CHITARRA, A. B. Pós-colheita de frutos e hortaliças: fisiologia e manuseio. 2. Ed. rev. e ampl. Lavras: UFLA, 2005.

DUARTE-ALMEIDA, J. M.; SANTOS, R. J.; GENOVESE, M. I.; LAJOLO, F. M. Avaliação da atividade antioxidante utilizando sistema $\beta$-caroteno/ácido linoléico e método de sequestro de radicais DPPH•. Ciência e Tecnologia de Alimentos, v. 26, n. 2, p. 446-452, 2006.

FAO (Food and Agriculture Organization). FAOSTAT. Disponível em: http://www.fao.org/waicent/portal/statistics. Acesso em: 18 de abril de 2014.

FERREIRA, M. F. P.; PENA, R. S. Estudo da secagem da casca do maracujá amarelo. Revista Brasileira de Produtos Agroindustriais, Campina Grande, v. 12, n. 1, p. 15$28,2010$.

HAIDA, K. S.; BARON, A.; HAIDA, K. S.; FACI, D.; HAAS, J.; SILVA, F. J. Compostos fenólicos totais e atividade antioxidante de duas variedades de goiaba e arruda. Revista Brasileira de Ciências da Saúde, ano 9, nº 28, abr/jun 2011.

HASSIMOTTO, N.M.A.; GENOVESE, M.I.; LAJOLO, F.M. Antioxidant activity of dietary fruits, vegetables, and commercial frozen fruit pulps, Journal of Agricultural and Food Chemistry, Chicago, v.53, n.8, p.2928-2935, 2005.

HIANE, P. A.; BOGO, D.; RAMOS, M. I. L.; RAMOS FILHO, M. M. Carotenoides próvitamínicos a e composição em ácidos graxos do fruto e da farinha do bacuri (Scheelea 
phalerata Mart.). Ciência e tecnologia de alimentos. Campinas, 23 (2): 206 - 209, maio ago. 2003.

INFANTE, J.; SELANI, M. M.; TOLEDO, N. M. V.; SILVEIRA-DINIZ, M. F.; ALENCAR, S. M.; SPOTO, M. H. F. Antioxidant activity of agroindustrial residues from tropical fruits. Alim. Nutr.= Braz. J. Food Nutr., Araraquara, v. 24, n. 1, p. 87-91, jan./mar. 2013.

LICHTENTHALER, H. and BUSCHMANN, C. 2001.Chlorophylls and carotenoids: Measurement and characterization by UV-VIS spectroscopy. Curr. Protoc. Food Anal. Chem. F 4.3.1-F 4.3.8. doi: 10.1002/0471142913.faf0403s01.

MAKRIS, D.P.; BOSKOU, G.; ANDRIKOPOULOS, N.K. Polyphenolic content and in vitro antioxidant characteristics of wine industry and other agri-food solid waste extracts. Journal of Food Composition and Analysis, San Diego, v. 20, p. 125-132, Mar., 2007.

MELO, E.A.; MACIEL, M.I.S.; LIMA, V.A.G.L.; NASCIMENTO, R.J. Capacidade antioxidante de frutas. Revista Brasileira de Ciências Farmacêuticas, São Paulo, v. 44, n.2, p. 193-201, 2008.

MELO, P.S.; BERGAMASCHI, K.B.; TIVERON, A.P.; ADNA PRADO MASSARIOLI, A.P.; OLDONI, T.L.C.; ZANUS, M.C.; PEREIRA, G.E.; ALENCAR, S.M. Composição fenólica e atividade antioxidante de resíduos agroindustriais. Ciência Rural, v.41, n.6, p.1088$1093,2011$.

NÓBREGA, E. M.; OLIVEIRA, E. L.; GENOVESE, M. I.; CORREIA, R. T. P. The impact of hot air drying on the physical-chemical characteristics, bioactive compounds and antioxidant activity of acerola (malphigia emarginata) residue. Journal of Food Processing and Preservation ISSN 1745-4549. 2014.

OLIVEIRA, R. GODOY, H.; PRADO, M. Otimização de metodologia colorimétrica para a determinação de ácido ascórbico em geleia de frutas. Ciência e Tecnologia de Alimentos, v. 30(1), p. 244-249, 2010.

PARK, K. J.; ANTONIO, G. C.; OLIVEIRA, R. A.; PARK, K. J. B. Conceitos de processo e equipamento de secagem. http://www.feagri.unicamp.br/ctea/projpesq.html. Campinas, Março de 2007.

PERAZZINI, H. Secagem de resíduos cítricos em secador rotativo. São Carlos, 2011. 104 f. Dissertação (Engenharia Química) - Universidade Federal de São Carlos, 2011.

PRETTE, A. P. Aproveitamento de polpa e resíduos de jaca (Artocarpus heterophyllus Lam.) através de secagem convectiva. Campina Grande, 2012. 161 f. Tese (Engenharia Agrícola) Universidade Federal de Campina Grande, 2012.

ROCHA, W.S.; LOPES, R. M.; SILVA, D. B.; VIEIRA, R. F.; SILVA, J. P.; AGOSTINECOSTA, T.S. Compostos fenólicos totais e taninos condensados em frutas nativas do cerrado. Rev. Bras. Frutic. Jaboticabal - SP, v. 33, n. 4, p. 1215-1221, Dezembro 2011. 
RODRIGUEZ-AMAYA, D. B.; KIMURA, M.; AMAYA-FARFAN, J. Fontes de carotenóides: tabela brasileira de composição de carotenoides em alimentos. Brasília: Ministério de Meio Ambiente/Secretaria de Biodiversidade e Florestas, 2008. 99 p.

RORIZ, R. F. C. Aproveitamento dos resíduos alimentícios obtidos das centrais de abastecimento do estado de Goiás S/A para alimentação humana. Goiânia, 2012. 162 f. Dissertação (Ciência e Tecnologia de Alimentos) - Universidade Federal de Goiás, 2012.

ROY, M.K.; TAKENAKA, M.; ISOBE, S.; TSUSHIDA, T. Antioxidant potential, antiproliferative activities, and phenolic content in water-soluble fractions of some commonly consumed vegetables: effects of thermal treatment. Food Chem., v.103, n.1, p.106-114, 2007.

SINGH, R. P.; HELDMAN, D. R. Introduction to food engineering. 3 th ed. San Diego: Academic, 2001. 659p.

SOUZA FILHO, M.S.M.; ARAGÃO, A.O.; ALVES, R.E.; FILGUEIRAS, H.A.C.F. Aspectos da colheita, pós-colheita e transformação industrial do pedúnculo do caju (Anacardium occidentale L.). Embrapa, 2000. Disponível em $<$ http://www.agencia.cnptia.embrapa.br/Repositorio/aspectoColheita_Caju_000g7d94 xb102wx5ok0wtedtxxfvd00.pdf>. Acesso em 19 de abril de 2014.

TANAKA, D. L. Influência da desidratação por spray drying Sobre o teor ácido ascórbico no suco de Acerola (malpighia ssp). 2007. 56f. Dissertação (Mestrado em Ciência dos Alimentos) - Centro de Ciências dos Alimentos, Departamento de Ciências, Programa de Pós-Graduação em alimentos e Nutrição, Universidade Estadual Paulista "Professor Júlio de Mesquita Filho", Araraquara.

TORALLES, R. P.; VENDRUSCOLO, J. L.; VENDRUSCOLO, C. T.; DEL PINO, F. A. B.; ANTUNES, P. L. Determinação das constantes cinéticas de degradação do ácido ascórbico em purê de pêssego: efeito da temperatura e concentração. Ciênc. Tecnol. Aliment., Campinas, 28(1): 18-23, jan.-mar. 2008.

VALDÉS, S. T.; VAZ TOSTES, M. G.; DELlA LUCIA, C. M.; HAMACEK, F. R.; PINHEIRO-SANT'ANA, H. M. Ácido ascórbico, carotenoides, fenólicos totais e atividade antioxidante em sucos industrializados e comercializados em diferentes embalagens. Rev. Inst. Adolfo Lutz. São Paulo, 2012; 71(4): 662-69.

VINHA, A. F.; ALVES, R. C.; BARREIRA, S. V. P.; CASTRO, A.; COSTA, A. S. G.; OLIVEIRA, M. B. P. P. Effect of peel and seed removal on the nutritional value and antioxidant activity of tomato ( Lycopersicon esculentum L.) fruits. LW T - Food Science and Technology 55 (2014) 197 e 202.

ZERDINA, K.; ROONEYA, M. L.; VERMUE, J. The vitamin C content of orange juice packed in an oxygen scavenger material. Food Chemistry 82 (2003) 387-395. 